\title{
Density inhomogeneity in ferroelectric thin films
}

\author{
Jiang-Li Cao, ${ }^{\text {a) }}$ Axel Solbach, and Uwe Klemradt \\ II. Physikalisches Institut B, RWTH Aachen University, Aachen 52056, Germany
}

Thomas Weirich and Joachim Mayer

Gemeinschaftslabor für Elektronenmikroskopie, RWTH Aachen University, Aachen 52074, Germany

Ulrich Böttger, Peter J. Schorn, and Rainer Waser

Institut für Werkstoffe der Elektrotechnik II, RWTH Aachen University, Aachen 52056, Germany

(Received 10 February 2006; accepted 15 June 2006; published online 31 July 2006)

\begin{abstract}
Structural investigations of $\mathrm{Pb}(\mathrm{Zr}, \mathrm{Ti}) \mathrm{O}_{3}$ (PZT) ferroelectric thin films derived by chemical solution deposition on $\mathrm{Pt} / \mathrm{TiO}_{x}$ electrode stacks were performed using grazing incidence $\mathrm{x}$-ray specular reflectivity of synchrotron radiation and transmission electron microscopy. A density inhomogeneity, i.e., a sublayer structure, in the PZT thin films was observed; the upper PZT sublayer had a lower density and the lower sublayer had a higher density. The influence of the density inhomogeneity, as a possible extrinsic contribution to size effects in ferroelectric thin films, was discussed. (C) 2006 American Institute of Physics. [DOI: 10.1063/1.2244045]
\end{abstract}

Ferroelectric thin films have received much attention owing to their promising applications in computer memory industries, micro-electro-mechanical systems, tunable microwave devices, etc. ${ }^{1,2}$ Nowadays, several very important topics related to the electrical properties of the low-dimensional ferroelectrics are not yet clear and need better understanding, including the size effects, ferroelectric fatigue, and imprint. As the electrical properties such as the dielectric and ferroelectric properties of ferroelectric materials are very sensitive to and dependent on the boundary conditions, thorough structural investigations of ferroelectric thin films are of crucial importance for an in-depth understanding of the topics mentioned above.

In all cases, the deposition method and growth conditions will affect the properties of the devices largely. Various physical deposition techniques such as molecular beam epitaxy (MBE), ${ }^{3}$ magnetron ${ }^{4}$ and ion-beam ${ }^{5}$ sputter deposition, and pulsed laser deposition (PLD), ${ }^{6}$ as well as chemical deposition including chemical vapor deposition ${ }^{7}$ and chemical solution deposition (CSD), ${ }^{8}$ have been used for the synthesis of ferroelectric thin films. Among these techniques, CSD has been widely used for the preparation of a variety of ceramic thin films of perovskite $\left(A B \mathrm{O}_{3}\right)$ structure, such as $\mathrm{BaTiO}_{3}, \mathrm{~Pb}(\mathrm{Zr}, \mathrm{Ti}) \mathrm{O}_{3},(\mathrm{Ba}, \mathrm{Sr}) \mathrm{TiO}_{3}, \mathrm{SrBi}_{2} \mathrm{Ta}_{2} \mathrm{O}_{9}$, etc., due to its high flexibility and low cost. ${ }^{9}$

In the present study, complementary analytical techniques of grazing incidence x-ray specular reflectivity (XRR) of synchrotron radiation and transmission electron microscopy (TEM) were used for the structural investigations of $\mathrm{Pb}(\mathrm{Zr}, \mathrm{Ti}) \mathrm{O}_{3}$ (PZT) thin films derived by $\mathrm{CSD}$ on $\mathrm{Pt} / \mathrm{TiO}_{x}$ electrodes. It is worth mentioning that XRR allows for the characterizations of electron density profile perpendicular to the sample surface on the depth scale from atomic dimension to $1 \mu \mathrm{m}$ depending on the wavelength of $\mathrm{x}$ rays and the composition of the materials. Among the techniques for studies of thin films, XRR owns some unique advantages such as high spatial sensitivity with a roughness and thickness resolution smaller than $0.1 \mathrm{~nm}$, high penetration power which

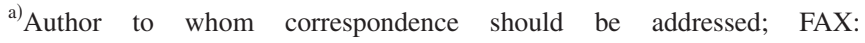
00492418022306; electronic mail: cao@physik.rwth-aachen.de allows for the observations in special environments such as protective atmosphere, etc., and nondestructive observations (only the x-ray ionization effect may be considered) ${ }^{10}$ However, XRR has received little attention for detailed structural analyses of ferroelectric thin films. In this letter, we will demonstrate that even a sublayer structure with a minute density difference in a single PZT thin film can be probed by XRR.

The $\mathrm{Pb}\left(\mathrm{Zr}_{0.3} \mathrm{Ti}_{0.7}\right) \mathrm{O}_{3}$ thin film was deposited using CSD on a $\mathrm{Pt} / \mathrm{TiO}_{x}$-electroded $\mathrm{Si}$ wafer with a size of $1 \times 1$ in. $^{211,12}$ After the spin coating of each three layers the sample was crystallized at $700{ }^{\circ} \mathrm{C}$ in oxygen for $5 \mathrm{~min}$ using a rapid thermal annealing (RTA) oven. It is compulsory to repeat this procedure until the PZT film reaches its intended thickness, in order to relax the stress in the ceramic film during the fabrication process, which is typical for a repetition deposition process. In this study, a PZT thin film of six layers was deposited. Then a stripe of Pt layer with a thickness of about $50 \mathrm{~nm}$ and a width of $2.5 \mathrm{~mm}$ was sputtered across the center of the PZT film. Subsequently, the sample was postannealed at $700{ }^{\circ} \mathrm{C}$ in oxygen for $5 \mathrm{~min}$ using RTA to remove the sputter damage at the top Pt/PZT interface caused by the ion bombardment during the Pt deposition. ${ }^{13}$ Finally, a long, narrow slice of the sample with a width of $4 \mathrm{~mm}$ and perpendicular to the Pt stripe was cut out from the $\mathrm{Si}$ substrate. Thus, a stripe of bare PZT thin film with a Pt-electroded capacitor in the center was obtained. The Pt-electroded capacitor was designated as the PZT capacitor, while a bare PZT area next to the capacitor was designated as the PZT thin film. XRR measurements with these two samples were carried out on a high-resolution reflectometer using monochromatic synchrotron radiation from Hasylab E2 (DESY) at a wavelength of $1.127 \AA$. Details of the experimental method and data processing can be found elsewhere. ${ }^{11,12}$ TEM observations were performed using a Philips Tecnai FEI 20. The thin TEM cross-sectional lamella was cut out from a PZT capacitor in the same batch by focused ion beam (FIB) using an FEI STRATA FIB 205.

Figure 1 shows the $x$-ray specular reflectivity of the PZT thin film and the PZT capacitor on a logarithmic scale as a function of the momentum transfer perpendicular to the 


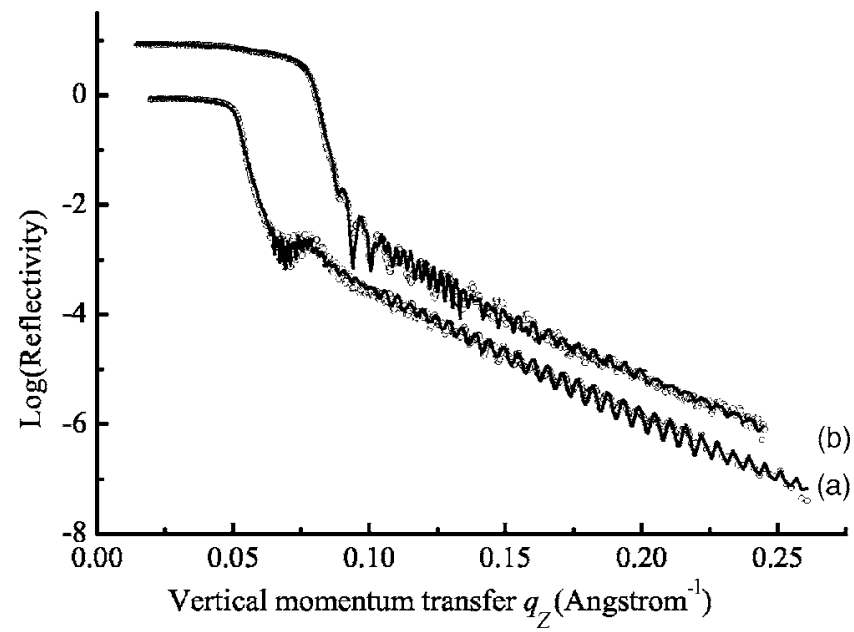

FIG. 1. Experimental x-ray specular reflectivities (open circles) of (a) the PZT thin film and (b) the PZT capacitor, and best fits (solid line) using the restricted homogenous stratified structure models of low-density PZT/highdensity $\mathrm{PZT} / \mathrm{Pt} / \mathrm{TiO}_{x}$ and $\mathrm{Pt} /$ low-density $\mathrm{PZT} /$ high-density $\mathrm{PZT} / \mathrm{Pt} / \mathrm{TiO}_{x}$, respectively.

sample surface, $q_{z}$. The experimental reflectivity curves are fitted, as also shown in Fig. 1. From the fits, a density inhomogeneity in the PZT layer is observed. The densities of 7.80 and $8.00 \mathrm{~g} / \mathrm{cm}^{3}$ for the upper and lower PZT sublayers, respectively, are derived. These two density values correspond to $97.1 \%$ and $99.6 \%$ of the theoretical density of $8.03 \mathrm{~g} / \mathrm{cm}^{3}$ calculated using the lattice parameters reported earlier. ${ }^{14}$ The structural characteristics obtained from best fits to the experimental reflectivity curves are summarized in Table I, in which the error margins are given for the indication of the accuracy. Here, for simplicity, $\mathrm{Ti}_{8} \mathrm{O}_{15}$ is used as the substrate, instead of $\mathrm{TiO}_{x},{ }^{11}$ because the reflectivity is insensitive to the deeper $\mathrm{SiO}_{2}$ layer.

It is noted that the top Pt/PZT interface with a roughness of $17.0 \AA$ is much smoother than the PZT surface with a roughness of $36.5 \AA$ through XRR measurements. This smoothening effect should originate from the bombardment

TABLE I. Thickness $(d)$, density $(\rho)$, and surface or interface roughness $(\sigma)$ of the constituent layers in the PZT thin film and the PZT capacitor obtained from XRR.

\begin{tabular}{|c|c|c|c|}
\hline $\begin{array}{c}\text { Constituent } \\
\text { layers }\end{array}$ & $\begin{array}{c}\text { Structure } \\
\text { parameters }\end{array}$ & $\begin{array}{l}\text { PZT thin } \\
\text { film }\end{array}$ & $\begin{array}{c}\text { PZT } \\
\text { capacitor }\end{array}$ \\
\hline \multirow[t]{3}{*}{ Top Pt } & $\sigma(\AA)$ & $\ldots$ & $36 \pm 1$ \\
\hline & $\rho\left(\mathrm{g} / \mathrm{cm}^{3}\right)$ & $\ldots$ & $21.1 \pm 0.1$ \\
\hline & $d(\AA)$ & $\cdots$ & $513 \pm 5$ \\
\hline \multirow[t]{3}{*}{ Upper PZT } & $\sigma(\AA)$ & $36.5 \pm 0.5$ & $17.0 \pm 0.5$ \\
\hline & $\rho\left(\mathrm{g} / \mathrm{cm}^{3}\right)$ & $7.80 \pm 0.02$ & $7.80 \pm 0.02$ \\
\hline & $d(\AA)$ & $964 \pm 5$ & $954 \pm 5$ \\
\hline \multirow[t]{3}{*}{ Lower PZT } & $\sigma(\AA)$ & $5 \pm 2$ & $5 \pm 2$ \\
\hline & $\rho\left(\mathrm{g} / \mathrm{cm}^{3}\right)$ & $8.00 \pm 0.03$ & $8.00 \pm 0.03$ \\
\hline & $d(\AA)$ & $1102 \pm 5$ & $1100 \pm 5$ \\
\hline \multirow[t]{3}{*}{ Bottom Pt } & $\sigma(\AA)$ & $12.5 \pm 0.3$ & $11.0 \pm 0.3$ \\
\hline & $\rho\left(\mathrm{g} / \mathrm{cm}^{3}\right)$ & $20.2 \pm 0.2$ & $20.2 \pm 0.5$ \\
\hline & $d(\AA)$ & $1016 \pm 3$ & $1013 \pm 3$ \\
\hline \multirow[t]{2}{*}{$\mathrm{TiO}_{x}$ layer } & $\sigma(\AA)$ & $9.5 \pm 0.3$ & $9.5 \pm 0.3$ \\
\hline & $\rho\left(\mathrm{g} / \mathrm{cm}^{3}\right)$ & 4.2 & 4.2 \\
\hline
\end{tabular}

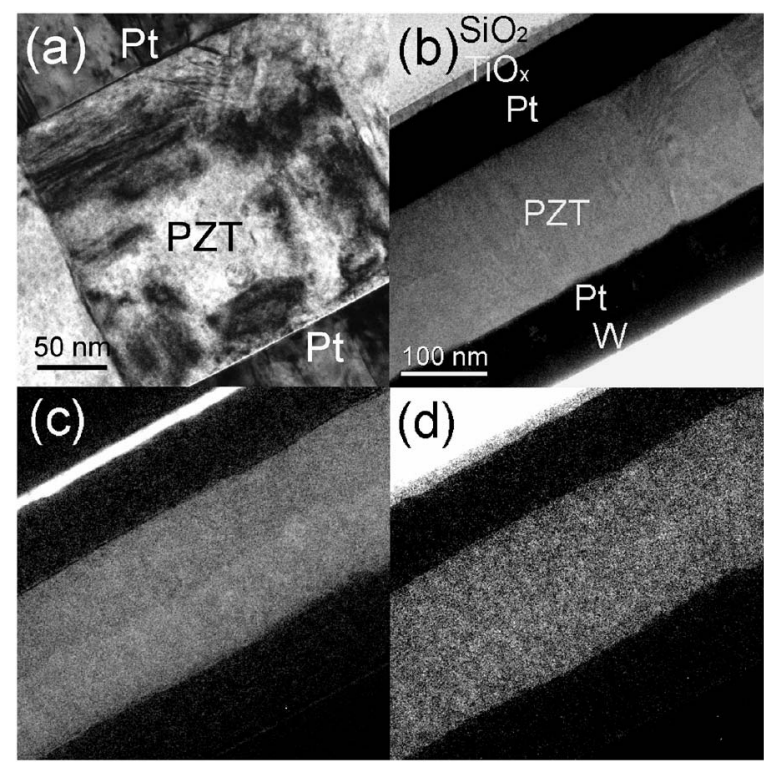

FIG. 2. Bright field cross-sectional TEM images of (a) a columnar PZT grain and (b) the multilayered structure of the PZT capacitor, and energy filtering images of (c) $\mathrm{Ti}$ and (d) $\mathrm{O}$.

of Pt ions or particles during the Pt deposition. Under the ion bombardment, the PZT surface is etched and a defect layer may be formed. Actually, the sputter damage on PZT thin films has been electrically observed and a postannealing can remove its influences. ${ }^{13}$ Very recently, Li et al. reported that they observed an individual interfacial layer at the $\mathrm{Pt} / \mathrm{BaTiO}_{3}$ interface by XRR using $\mathrm{x}$ rays of tube source, which they suggested to be a dead layer in ferroelectric thin films. ${ }^{15}$ However, it is difficult to make a comparison between our results because the details for the preparation of their Pt top electrode and any postannealing were not given.

Figure 2(a) shows a bright field cross-sectional TEM image of a PZT capacitor, in which the columnar grain structure in the PZT thin film is depicted. Figure 2(b) gives a bright field cross-sectional TEM image with a lower magnification. From top left to bottom right is the $\mathrm{SiO}_{2}, \mathrm{TiO}_{x}$, Pt bottom electrode, PZT, and Pt top electrode as well as the $\mathrm{W}$ layer deposited for the FIB cutting. About half of the PZT layer in the thickness direction next to the Pt top electrode is brighter than the other half, though the contrast is weak as it is. The contrast in the PZT layer indicates that there are two sublayers in the PZT film. In the bright field image, the darkness is proportional to the electron density for the case under study. Therefore, the PZT sublayer next to the Pt bottom electrode has a higher electron density, which agrees well with the XRR results. Similarly, the contrast can be also observed in the energy filtering images of Ti and $\mathrm{O}$, as given in Figs. 2(c) and 2(d), respectively. Though there are limited element diffusions as we observed previously using scanning transmission electron microsopy, ${ }^{11}$ the multilayered structure can be seen clearly and the ferroelectric/electrode interfaces are very clear.

Size effects in ferroelectric materials have been extensively studied for several decades, but still remain poorly understood regarding the critical size and origins. However, the critical size of ferroelectric that is either theoretically predicted or experimentally observed has been always decreasing as time passes by ${ }^{16,17}$ Normally, the intrinsic contribution to the size effects is referred to the general spatial
to AIP license or copyright, see http://apl.aip.org/apl/copyright.jsp 
correlations of the ferroelectric polarization of electrostatic origin. ${ }^{18}$ The extrinsic size effects may originate from a nonferroelectric dead layer at the interfaces caused by the chemistry inhomogeneity ${ }^{19}$ or nearby-electrode charge injection, ${ }^{20}$ the depolarizing field caused by the nonideally conductive electrode, ${ }^{21}$ the electrostrictive constraint ${ }^{22}$ and lattice mismatch at the ferroelectric/electrode interface, the growth stress which may be compressive or tensile, ${ }^{16}$ and the Schottky depletion layer caused by the mismatch in the band structure at the interfaces. ${ }^{23}$ Size effects in ferroelectric capacitors are complicated since the parameters are characteristic of not only the ferroelectric, but also of a system including two electrodes. A dead layer mechanism has been frequently employed to explain qualitatively the size effects and fatigue ${ }^{20}$ or imprint. ${ }^{24}$ However, the possible extrinsic contribution of the density inhomogeneity to the size effects needs to be emphasized.

The columnar grains in the PZT thin film consist of two parts, i.e., one with a higher density next to the Pt bottom electrode and the other one with a lower density on top. The density inhomogeneity in the PZT film can be attributed to the growth mode and the thermal history difference, as explained in the following. For chemical solution deposition of the first three layers of PZT, the nucleation occurs at the interface of PZT/bottom Pt upon the first crystallization other than in the PZT layer randomly, which answers for the columnar structure of the PZT grains. For the deposition of the following three layers of PZT, the nucleation takes place on the surface of the lower PZT ceramic layer upon the second crystallization, and then, essentially, an epitaxial growth follows. Thus, the two sublayers have different growth modes. Moreover, the first PZT ceramic sublayer received $5 \mathrm{~min}$ thermal annealing more than the second PZT sublayer. Although the two sublayers have actually the same $\mathrm{Pb}: \mathrm{Zr}: \mathrm{Ti}$ ratio, the content of the residual impurities and crystallinity may be different, as indicated by previous results. ${ }^{11}$

The upper PZT sublayer (or sublayers if more than two crystallizations are applied for the film preparation) may have different electrical properties from those of the lower sublayer. Indeed, it was observed that the dielectric constant increases due to the further densification of the PZT ceramic with increasing annealing time. ${ }^{12}$ Thus, the whole PZT film derived by CSD can be regarded as the sublayers in series. By decreasing the PZT thickness of the relatively thick films and hence the numbers of thermal annealing for the lower ceramic sublayers, the dielectric constant would decrease, which constitutes an extrinsic size effect. Therefore, it is necessary to have samples with the same structure and boundary conditions to ensure that the measured effects do reflect the intrinsic size effects. This discussion applies not only to the ferroelectric thin films derived by CSD, a repetition process, but also to those derived by a one-step continuous deposition technique in which the deposits are crystallized after they are condensed on the high-temperature substrate, such as PLD,
MBE, etc. Therefore, a density gradient, rather than a density step, may form under a thermal history gradient. Currently, this implication is tentative and more experimental investigations are needed.

In summary, XRR of synchrotron radiation and TEM were used for the structural investigations of PZT thin films derived by CSD. The entire capacitor structure with a Pt top electrode and a relatively thick PZT ceramic film can be seen by the highly parallel $\mathrm{x}$ rays of synchrotron radiation, while the structural parameters of the constituent layers in the capacitor were obtained under nondestructive conditions. The density inhomogeneity in the PZT thin films was observed, and its influence on the size effects was discussed.

One of the authors (J.-L.C.) thanks the Alexander von Humboldt Foundation for support to this Humboldt project. The authors thank DESY for Grant No. II-03-009.

${ }^{1}$ J. F. Scott and C. A. Paz de Araujo, Science 246, 1400 (1989).

${ }^{2}$ O. Auciello, J. F. Scott, and R. Ramesh, Phys. Today 51(7), 22 (1998).

${ }^{3}$ T. Zhao, F. Chen, H. Lu, G. Yang, and Z. Chen, J. Appl. Phys. 87, 7442 (2000).

${ }^{4}$ S. H. Seo, H. C. Kang, D. Y. Noh, Y. Yamada, and K. Wasa, Appl. Phys. Lett. 84, 3133 (2004).

${ }^{5}$ S. B. Krupanidhi, H. Hu, and V. Kumar, J. Appl. Phys. 71, 376 (1992).

${ }^{6}$ V. Nagarajan, A. Roytburd, A. Stanishevsky, S. Prasertchoung, T. Zhao, L. Chen, J. Melngailis, O. Auciello, and R. Ramesh, Nat. Mater. 2, 43 (2003).

${ }^{7}$ C. M. Foster, Z. Li, M. Buckett, D. Miller, P. M. Baldo, L. E. Rehn, G. R. Bai, D. Guo, H. You, and K. L. Merkle, J. Appl. Phys. 78, 2607 (1995).

${ }^{8}$ R. Waser, T. Schneller, S. Hoffmann-Eifert, and P. Ehrhart, Integr. Ferroelectr. 36, 3 (2001).

${ }^{9}$ R. W. Schwartz, T. Schneller, and R. Waser, C. R. Chim. 7, 433 (2004).

${ }^{10}$ E. Chason and T. M. Mayer, Crit. Rev. Solid State Mater. Sci. 22, 1 (1997).

${ }^{11}$ J. L. Cao, A. Solbach, U. Klemradt, T. Weirich, J. Mayer, U. Boettger, U. Ellerkmann, P. J. Schorn, P. Gerber, and R. Waser, J. Am. Ceram. Soc. 89, 1321 (2006).

${ }^{12}$ J. L. Cao, A. Solbach, U. Klemradt, U. Boettger, U. Ellerkmann, P. J. Schorn, P. Gerber, and R. Waser (unpublished).

${ }^{13}$ U. Ellerkmann, P. Schorn, D. Bolten, U. Boettger, R. Waser, R. Bruchhaus, and K. Yamakawa, Integr. Ferroelectr. 52, 63 (2003).

${ }^{14}$ N. Tohge, S. Takahashi, and T. Minami, J. Am. Ceram. Soc. 74, 67 (1991).

${ }^{15}$ X. L. Li, B. Chen, H. Y. Jing, H. B. Lu, B. R. Zhao, Z. H. Mai, and Q. J. Jia, Appl. Phys. Lett. 87, 222905 (2005).

${ }^{16}$ H. Kohlstedt, N. A. Pertsev, and R. Waser, Mater. Res. Soc. Symp. Proc. 688, C6.5.1 (2002).

${ }^{17}$ D. D. Fong, G. B. Stephenson, S. K. Streiffer, J. A. Eastman, O. Auciello, P. H. Fuoss, and C. Thompson, Science 304, 1650 (2004).

${ }^{18}$ R. Kretschmer and K. Binder, Phys. Rev. B 20, 1065 (1979).

${ }^{19}$ P. K. Larsen, G. J. M. Dormans, D. J. Taylor, and P. J. van Veldhoven, J. Appl. Phys. 76, 2405 (1994).

${ }^{20}$ A. K. Tagantsev, I. Stolichnov, E. L. Colla, and N. Setter, J. Appl. Phys. 90, 1387 (2001)

${ }^{21}$ J. Junquera and P. Ghosez, Nature (London) 422, 506 (2003).

${ }^{22}$ Z. Suo, Curr. Opin. Solid State Mater. Sci. 3, 486 (1998).

${ }^{23}$ J. Robertson and C. W. Chen, Appl. Phys. Lett. 74, 1168 (1999).

${ }^{24}$ M. Alexe, C. Harnagea, D. Hesse, and U. Goesele, Appl. Phys. Lett. 79, 242 (2001). 